\title{
Entre usage professionnel des TIC et structure organisationnelle : la capacité au bricolage comme compétence adaptative
}

ICT Use and Organizational Structure: Knowing How to Tinker as an Adaptive Skill

Isabelle Comtet

\section{OpenEdition}

Journals

Édition électronique

URL : http://journals.openedition.org/edc/1079

DOl : 10.4000/edc. 1079

ISSN : 2101-0366

Éditeur

Université Lille-3

Édition imprimée

Date de publication : 1 décembre 2009

Pagination : 119-134

ISSN : 1270-6841

Référence électronique

Isabelle Comtet, «Entre usage professionnel des TIC et structure organisationnelle : la capacité au bricolage comme compétence adaptative », Études de communication [En ligne], 33 | 2009, mis en ligne le 01 décembre 2011, consulté le 01 mai 2019. URL : http://journals.openedition.org/edc/1079 DOI : 10.4000/edc. 1079

Ce document a été généré automatiquement le 1 mai 2019.

(C) Tous droits réservés 


\title{
Entre usage professionnel des TIC et structure organisationnelle : la capacité au bricolage comme compétence adaptative
}

\author{
ICT Use and Organizational Structure: Knowing How to Tinker as an Adaptive \\ Skill
}

Isabelle Comtet

\section{Introduction}

1 La mise en œuvre de TIC au sein des organisations implique, les études empiriques l'ont souligné, de réfléchir au processus global d'implémentation. Cette dernière consiste à réaliser la phase finale d'élaboration d'un système (d'information ou non) qui permet au matériel, aux logiciels et aux procédures d'entrer en fonction de façon optimale. Ainsi, ce qui est en jeu, c'est l'utilisation efficace des technologies, du point de vue des utilisateurs comme du point de vue des organisations. Ce questionnement s'inscrit dans la lignée des recherches menées sur l'informatisation des organisations et des échanges. Le développement $d u$ processus d'informatisation au cours des vingts dernières années, a généré ou révélé un certain nombre de changements profonds au sein des organisations, qui touchent aujourd'hui tout autant la vie interne des organisations, les relations entre les différents marchés et les pratiques des acteurs que la façon de réfléchir et de conceptualiser les phénomènes organisationnels et économiques (Benghozi, 2002). L'emploi des TIC est désormais considéré comme un élément fondamental de l'efficacité des entreprises, et notamment parce qu'elles améliorent les procédures de contrôle (homogénéité, cohérence, vérification de la qualité), développent la flexibilité des organisations (synchronisme, asynchronisme, réactivité, réduction des distances) et favorisent une mise en synergie de compétences spécifiques. 
2 Pour l'implémentation d'un dispositif, donc afin d'interroger le fonctionnement de celuici, il est pour nous central, de mettre en perspective la structure organisationnelle et la dynamique dans lesquelles s'insèrent les TIC et l'activité de l'individu. Nous ne voulons pas ici proposer une revue exhaustive de la littérature sur la dialectique entre structure organisationnelle et mise en œuvre des TIC (à l'instar de nombreux chercheurs depuis une vingtaine d'années, notamment ceux issus du courant structurationniste) nous nous focalisons sur la marge de liberté « laissée » aux acteurs pour s'approprier un dispositif sociotechnique dans la mise en œuvre réussie (c'est-à-dire efficace) de TIC au sein d'une organisation. Notre cadre théorique est donc celui de la littérature structurationniste: elle nous permet de comprendre (notamment au travers des apports d'auteurs comme de Sanctis, Poole ou Orlikowski) dans quelle mesure la structuration d'une activité permet ou non - aux acteurs d'avoir une autonomie de «bricolage » des TIC qui semble nécessaire à l'appropriation du dispositif sociotechnique. Nous envisageons ainsi le bricolage non seulement comme une phase de l'appropriation des TIC par les usagers, mais comme une compétence à part entière chez ceux-ci au fil de l'évolution permanente des dispositifs technologiques.

3 Notre travail repose sur deux hypothèses imbriquées.

4 La première est plus « méso » et met en avant l'appropriation des systèmes d'information, dans le cadre d'une activité professionnelle. Elle postule l'efficacité de l'usage des SI quand l'organisation laisse aux acteurs la possibilité de détourner l'usage de ces systèmes.

5 La seconde est plus "micro»: l'appropriation réussie (donc efficace) d'un nouveau dispositif sociotechnique implique une phase de bricolage pendant laquelle l'acteur manipule la technologie (à l'aide d'éléments stockés à cette fin ou non) pour la rendre conforme à ses attentes et à ses besoins professionnels.

6 Afin de vérifier ces hypothèses, nous avons mené une enquête qualitative (questionnaire semi-directif en ligne) dont la cible est constituée de 128 salariés d'entreprises (de tous secteurs et de toutes activités) quelles que soient leurs fonctions. Cette enquête a débuté au mois de mai 2008 et le recueil des données s'est effectué jusqu'au mois de septembre 2008.

\section{Interactions entre structures d'activité professionnelle et pratiques sociales}

7 La réalité sociale de l'usage de TIC est duale dans le sens où elle mêle dimensions objectives et subjectives de l'acteur et du système. Ainsi, la théorie de la structuration met en avant différents points clés essentiels à notre réflexion (Clark, Modgil \& Modgil, 1990) : elle souligne les pratiques sociales qui se trouvent au fondement de la constitution des individus et de la société. Dès lors, en reproduisant des pratiques sociales issues de la vie en société, les acteurs établissent les règles et la structure des dispositifs de cette dernière. Ainsi, la structure est dépendante de l'activité : c'est en même temps le moyen et le résultat d'un processus de structuration que Giddens nomme double herméneutique " we create society at the same time as we are created by it ». Ainsi, les interactions entre structure et acteurs revêtent plusieurs dimensions: celle de la communication interpersonnelle qui permet à chacun de donner du sens aux actions. Celle du pouvoir qui, par utilisation de moyens matériels ou non, autorise ou contraint la production et la reproduction de structure de domination lors des interactions. Celle des valeurs qui 
permettent aux acteurs de contrôler et sanctionner leurs actions en fonction de normes de valeurs.

8 A travers le prisme du structurationnisme (De Sanctis \& Poole, 1994), les TIC et les systèmes d'information de façon plus générale (S.I.) sont considérés et conceptualisés comme le produit des actions humaines. Ces dernières en sont renouvelées et contraignent autant qu'elles permettent de telles productions.

\section{Le bricolage au cœur des pratiques et des usages des TIC}

9 Dans cette perspective, si l'on écoute attentivement les remarques des utilisateurs des TIC, notamment en entreprise, on entend régulièrement les phrases suivantes: "j'ai bricolé mon ordinateur pour avoir cette requête ", ou encore "j'ai bricolé sur l'ERP parce que je ne me souvenais pas comment on faisait », ou également «en bricolant le logiciel, tu pourrais faire la même chose que moi ». De même, les MOA (maîtres d'ouvrages) ou les informaticiens des SI en entreprise reprennent à leur compte ce langage : « mais qu'est-ce que tu as encore bricolé pour que ça ne marche pas?", "je vais te bricoler un accès » ou encore "de toute façon, vous bricolez tous vos ordinateurs et nous on vient pour éteindre le feu ». On constate ainsi que le bricolage semble d'une part fréquemment utilisé (en tant que pratique) dans les structures d'activité professionnelle, d'autre part qu'il se rapporte à une action que l'on n'attendait pas, c'est-à-dire qui n'est pas prévue dans les usages initiaux, mais que l'on sait existante.

\section{La notion de bricolage}

Nous insistons ici sur le fait que le terme de bricolage ne s'emploie évidemment pas uniquement dans le contexte d'usages de nouvelles technologies.

11 Cela dit, il peut être éclairant d'envisager la notion de bricolage dans son acception la plus quotidienne. Elle y est définie par l'action, l'habitude, et la réparation. D'une façon très banale, il s'agit « d'action, d'habitude de bricoler » puis de « réparation faite tant bien que mal ». Cette définition souligne d'abord ce que fait quelqu'un et ce par quoi il réalise une intention. Il y a donc ici l'idée essentielle de se proposer un certain but. La réalisation de ce dernier implique, de façon sous jacente, la volonté de réalisation.

12 Ensuite, cette définition met l'accent sur une manière de se comporter, d'agir, individuellement, de façon répétée. Le point important concerne ici l'action individuelle de façon réitérée. Il s'agit de reproduire une façon de faire.

13 Enfin, le dernier point de cette définition souligne un travail qui consiste à remettre en bon état. Cela sous entend que l'état initial n'est plus optimal, n'est plus (n'est pas ?) conforme à ce qu'il devrait être.

14 Ainsi, rapportée à la problématique d'usage des TIC, la notion de bricolage met en avant plusieurs éléments fondamentaux que l'on peut regrouper de la façon suivante : il s'agit de la volonté, individuelle et réitérée, de rendre conforme à l'objectif envisagé, l'usage d'un dispositif technique.

15 Si l'on essaie de regarder la notion de bricolage en mettant de côté une perspective uniquement utilitariste des TIC, on peut examiner le bricolage à travers l'acception 
originelle proposée par Lévi-Strauss (1962). Ce dernier le présente comme la science du concret (titre de son chapitre premier). Selon lui, les premiers hommes ou les peuples sauvages relèvent « d'une pensée première » qui traduit une intelligence pratique. Il y a là la faculté de connaître et de comprendre l'action, de transformer la réalité extérieure par la volonté humaine.

La notion de bricolage a été également utilisée par Ciborra qui a analysé le fossé existant entre la théorie de l'usage des TIC et la pratique du développement de ces dernières (Ciborra, 2004b). Les situations réelles d'usage supposent de prendre en compte ce qu'il appelle (en reprenant les mots de Lévi-Strauss) des pratiques d'improvisation et de débrouillage, c'est-à-dire de bricolage. C'est selon lui, une véritable stratégie de création (en l'occurrence, il parle précisément de la pratique du design de systèmes d'information) que d'utiliser le bricolage comme modèle pour développer la créativité des développeurs, résoudre des problèmes et trouver des solutions. On est ici dans l'optique d'outils adaptés à une structure. Mais le problème se pose également dans une perspective d'appropriation puis d'usage des TIC en général.

\section{Vers l'appropriation des TIC au sein des structures d'activité}

Nous retrouvons dans les éléments de définition de Lévi-strauss l'idée d'action, de volonté et de transformation d'un objet. Néanmoins, l'auteur ajoute deux facteurs qui paraissent essentiels à notre problématique: le premier fait référence à l'idée de faire "avec les moyens du bord». Le second souligne l'idée de stock. Il parle « d'un ensemble à chaque instant fini d'outils et de matériaux, hétéroclites au surplus, parce que la composition de l'ensemble n'est pas en rapport avec le projet du moment, ni d'ailleurs avec aucun projet particulier, mais le résultat contingent de toutes occasions qui se sont présentées de renouveler ou d'enrichir le stock ou de l'entretenir avec les résidus de construction et de destruction antérieurs ». Cette vision du bricolage suppose que l'utilisateur se sert de tout ce qui peut l'aider pour à arriver à ses fins. Il implique donc également une très bonne connaissance des éléments du stock qui serviront de moyens d'atteindre son objectif.

Dans cette optique, le bricolage peut servir à comprendre les situations d'action professionnelle dans la mesure où ces dernières sont porteuses de sens au niveau de la relation organisation - acteur - contexte. Trois raisons peuvent être évoquées :

- la première souligne l'obligation pour les acteurs en situation d'activité professionnelle d'innover pour rester efficaces. Face à un environnement social et économique peu prévisible où le contexte est le lieu d'une forte pression sociale, les acteurs mettent en œuvre les moyens de gérer l'incertitude. Le bricolage est une réponse qui peut être rapide et efficiente parce qu'elle est basée sur l'improvisation et l'immédiateté. Le bricolage est donc un moyen d'action réactif au sein de l'organisation.

- la seconde part du principe que le bricolage, comme moyen de s'approprier des dispositifs organisationnels, peut favoriser la mémoire organisationnelle. Celle-ci désigne l'ensemble des processus par lesquels les organisations traitent, conservent et réutilisent des informations. Les acteurs, en rendant propres à leurs utilisations, un dispositif, concourent à nourrir cette mémoire dont le rôle est central en termes de communication dans les processus de traitement de l'information au niveau collectif. La mémoire organisationnelle favoriserait ainsi la gestion des connaissances (knowledge management) en entreprise. Cette relation entre le bricolage et l'apprentissage comme canalisateur des connaissances développées collectivement au sein de l'entreprise est sous-jacente. 
- la troisième s'appuie sur le fait que le bricolage peut être utilisé comme clé pour la compréhension des usages en management des systèmes d'information dans la mesure où l'usage prescrit des dispositifs sociotechniques ne se révèle pas nécessairement être celui adopté par les utilisateurs. Cette idée, qui n'est pas récente, a été notamment développée par De Certeau (1990) et met en avant le lien existant entre usage prescrit et usage réel des dispositifs. Il insiste ainsi sur l'interaction entre acteur, dispositif et organisation comme moteur de l'action.

19 Dans une visée complémentaire, De Sanctis \& Poole et Orlikowski, établissent clairement que la technologie est un artefact physique avec des propriétés matérielles. Sa conception et son usage sont socialement construits. Dans cette perspective, le bricolage, tel que nous l'avons défini plus avant peut alors être pensé comme la possibilité laissée aux acteurs de s'approprier la structure (sociotechnologique) fournie par l'organisation. Cette autonomie donnée ou prise pour transformer la structure de façon à la rendre efficace (c'est-à-dire conforme aux attentes des acteurs pour atteindre un but) est le gage de l'appropriation et donc, in fine, de l'implémentation optimale des TIC. Le bricolage peut donc être considéré comme la possibilité que l'organisation donne ou laisse aux acteurs d'interagir avec la structure sociotechnologique.

\section{Principaux résultats de l'enquête}

20 L'enquête que nous avons menée en 2008, montre que la capacité à bricoler semble constituer une compétence clé pour la réalisation de l'activité professionnelle, en particulier dans le secteur tertiaire : cette compétence serait effectivement issue pour partie du fonctionnement institutionnel de l'entreprise dans laquelle les professionnels utilisent les systèmes d'information. En outre, elle répond à un besoin des salariés en termes de mise à jour des usages des SI et donc d'efficacité au travail.

\section{Contexte d'enquête}

21 La majorité des personnes interrogées (qui a souhaitée répondre au questionnaire) se trouve dans une tranche d'âge relativement jeune pour des professionnels (18/33 ans). Ces personnes ont donc une expérience de l'entreprise et des organisations assez courte. Cela implique, qu'il s'agit de personnes ayant peu changé de service, et connaissant donc assez bien le fonctionnement organisationnel (procédure de réalisations de travail sur les SI, possibilité de détournement d'usage etc..) de l'organisation dans laquelle elles sont. Cela dit, la tranche d'âge des 34/49 ans est également assez bien représentée.

En outre, la majorité des répondants se situe dans la catégorie des cadres moyens ( $58 \%$ des réponses). Une partie importante des interviewés est formée de « cadres » (40\%). Ces résultats sont à mettre en perspective avec les données classiques de l'Insee qui soulignent que les cadres supérieurs, mais également les cadres moyens, sont majoritairement des hommes. Ce qui explique la part plus importante de répondants « hommes » dans notre enquête.

Par ailleurs, hormis le pôle finance (Gestion financière, contrôle, comptabilité) et la R\&D, toutes les fonctions sont représentées. Néanmoins, la plupart des réponses ont été données par des salariés de la fonction Système d'information et de la fonction Direction générale. Ces deux fonctions ont un intérêt pour l'enquête : un intérêt en ce qui concerne 
les usages des systèmes d'information en entreprise pour la fonction Système d'information (notamment pour les personnes en fonction support ou en Assistance à Maitrise d'Ouvrage); un intérêt également en termes de pratiques managériales pour l'usage des systèmes d'information pour les salariés du service de direction générale.

Notre réserve sur notre échantillon est celle qui existe eu égard à la majorité des enquêtes dédiées aux usages des TIC via internet, impliquant une connaissances des évolutions de ces derniers, donc à ceux qui « pratiquent» le plus les outils au quotidien. En outre, les contraintes de temps et de moyens inhérents à l'enquête ont nécessité l'utilisation de questionnaires qualitatifs privilégiant le semi-discursif avec les limites que comporte cette approche, notamment par rapport à des méthodes plus expérimentales.

Ainsi, nous avons mis en évidence que les enjeux liés à la situation de travail (1) et les impératifs du fonctionnement organisationnel (2) poussent, implicitement les salariés à façonner par essais et erreurs les dispositifs techniques nécessaires à leur activité (3).

\section{Quant le contexte de travail pousse au bricolage}

Les contextes économique, financier mais également organisationnel et humain, incitent les entreprises, plus ou moins consciemment, à développer des situations de travail complexes pour les salariés: ces derniers doivent cependant dans un premier temps répondre à une obligation de résultat. Cela se traduit dans la majeure partie des entreprises par une mesure de l'atteinte des objectifs annuels et une mise à jour de ceuxlà pour l'année suivante. Dans le même temps, les professionnels doivent faire face à des délais de plus en plus courts : qu'il s'agisse de délais de production ou de service, ceux-ci sont essentiels à la compétitivité des entreprises. En raison du contexte, les professionnels sont donc contraints à aller plus vite, en maximisant la qualité. Dans cette recherche de compétitivité, les systèmes d'information jouent un rôle de premier plan. Ils permettent de raccourcir le délai de réponse, de multiplier les informations et d'accélérer leur traitement pour atteindre un seuil de qualité répondant aux exigences du marché.

Le fait de ne pas pouvoir utiliser les SI (pour quelques raisons que ce soit) annihile cette possibilité de compétitivité et contraint les professionnels à rechercher d'autres solutions. Soit ils utilisent un autre système, soient ils contournent la procédure. L'obligation pour certains usagers (la moitié des répondants) d'utiliser les SI définis par les procédures de travail «je suis obligée de me servir de cet outil, parce qu'il a été mis en place exprès pour nous. Mon chef ne comprendrait pas que je fasse autrement !» (Cadre dans la fonction marketing), ajoute encore aux contraintes de travail. Pour répondre à cette nécessité d'usage des SI dans toutes les situations, les utilisateurs n'ont alors guère le choix : ils bricolent ( $50 \%$ des cas). C'est-à-dire qu'ils remettent un outil défaillant en conformité avec leurs besoins.

28 Parallèlement à cela, l'organisation interne, le fonctionnement institutionnel favorisent également ce comportement.

\section{Quant le fonctionnement organisationnel incite au bricolage}

Toute activité professionnelle doit s'adapter à son environnement. Pour cela, les entreprises modifient annuellement les objectifs (de productivité) de leurs collaborateurs. Ainsi, chaque année, tous les professionnels voient leur charge de travail évoluer. Mais 
l'organisation, elle, n'évolue pas dans le même temps. Elle n'a ni le temps, ni les moyens de s'adapter aussi rapidement aux changements d'activité des salariés.

Or, il serait nécessaire dans ce cas d'apporter des modifications non seulement en termes de procédures de réalisation de la charge de travail, mais également en termes d'outils de travail. Sans transformer intégralement les systèmes d'information de l'entreprises, ceuxci doivent être adaptés aux modifications de réalisation de l'activité " on est censé utiliser le système pour notre travail, mais moi j'en utilise un autre qui marche plus vite.. "(Cadre en Ressources Humaines). Dans le même temps, si les SI sont modifiés ou si les professionnels en font d'autres usages (pour cause de modification des objectifs), un apprentissage s'impose. Néanmoins, la plupart du temps, les entreprises n'ont ni le temps matériel "Pour former, il faut une salle avec du matériel, et du temps. Ben, ça, on n'a pas. On aide les usagers sur les ordi directement » (cadre en SI), ni le budget pour former très régulièrement tous les salariés (toutes les formations menées par les répondants à notre échantillon remontent à plus de 2 ans) « on est formé quant on arrive, sur tout le logiciel. On se sert de $5 \%$, le reste on oublie et après on bidouille " (cadre en Marketing) pour implémenter les outils de cette façon.

31 Ainsi, les professionnels usagers des SI sont dans l'obligation de s'adapter par eux-mêmes au système et à son fonctionnement. Pour être efficaces, ils sont contraints, implicitement, par l'organisation, de bricoler les outils avec lesquels ils travaillent. Cette capacité d'adaptation devient alors une compétence clé.

\section{Le bricolage : une compétence clé, implicitement autorisée}

Dans la mesure où l'activité professionnelle évolue régulièrement, sans que les SI puissent s'adapter et/ou sans que l'organisation puisse répondre à un besoin de formation, les salariés sont bien dans l'obligation de mettre en œuvre une réappropriation régulière et permanente des outils qu'ils utilisent (97\% des répondants gèrent par eux-mêmes les défaillances de leur SI). Il ne s'agit plus seulement de s'approprier, mais d'improviser « de toute façon, il faut bien faire le travail. Si personne ne peut m'aider, mon chef s'en fiche que je n'y arrive pas. Faut être efficace quand même» (cadre en marketing), d'apprendre "avec ma collègue on s'aide. L'une sait toujours des trucs que l'autre ne sait pas, et on apprend comme ça " (cadre en supply chain), de créer de nouvelles fonctionnalités, ou de nouvelles procédures pour adapter le dispositif aux évolutions de leurs besoins en matière d'activité professionnelle. L'idée est d'arriver à une modification de l'outil lui-même, soit de façon individuelle, soit de façon "collective» au sens collaboratif du terme, c'est-à-dire impliquant des objectifs communs et des actions de modifications communes à plusieurs professionnels.

33 Il peut également s'agir de mettre en œuvre cette compétence pour arriver à «jouer » avec l'ensemble des outils à disposition des professionnels. Les entreprises (comme le soulignent certaines des réponses « « on est censé utiliser le système pour notre travail, mais moi j'en utilise un autre qui marche plus vite») proposent généralement un ensemble d'outils pour réaliser leur activité. La capacité d'un professionnel à changer d'instrument de travail rejoint l'idée selon laquelle l'usager doit posséder un stock d'éléments, déjà pour partie appropriés, qu'il choisit et met en œuvre pour rester efficace.

Cela implique que les usagers des SI entretiennent les compétences nécessaires pour toujours rester en adéquation avec leur poste, qu'ils sachent autogérer les difficultés 
d'usages, et les dépasser de façon à devenir quasi autonomes en termes de formation et d'appropriation du dispositif technique.

Ce type de compétence est d'autant plus à développer qu'elle est implicitement souhaitée et appréciée par le fonctionnement organisationnel de l'entreprise "on demande à des cadres d'être adaptables, souples et flexibles. Il faut qu'ils sachent gérer l'urgence avec les moyens qu'ils ont, en étant efficaces. C'est leur boulot à ce niveau" (responsable finance). En ce sens, l'entreprise "pousse» les acteurs à développer de nouveaux savoirs, de nouvelles compétences, qui vont bien au-delà du simple usage, de la simple appropriation des outils technologiques.

\section{Eléments de discussion}

Par rapport à la problématique, l'approche du structurationnisme nous permet de mettre l'accent sur deux points essentiels :

- le premier souligne l'importance de faire évoluer la technologie (dans ses fonctionnalités) en fonction du contexte d'action collectif. Il y a ici l'idée essentielle pour nous de détermination de l'action en fonction des éléments du contexte externe et interne, notamment en termes de groupe social. L'utilisation en situation fait qu'une technologie est différente selon les groupes sociaux dans lesquels elle est inscrite. Sa transformation (le bricolage qui l'affecte) répond à une appropriation différente selon les structures sociales. En se plaçant du point de vue des concepteurs, et non plus des usagers, (mais cela concourt à conforter notre hypothèse), Ciborra propose la notion "d'hospitalité » pour développer les systèmes d'information (Ciborra, 2004). L'hospitalité est avant tout comprise comme la possibilité d'accueillir un étranger chez soi. Transposée aux TIC, l'hospitalité implique que l'utilisateur final a la possibilité de «s'inviter » au sein du dispositif technique. Si ce dernier doit suivre les règles de l'hôte (le dispositif technique), il n'est pas pour autant obligé de se soumettre à sa culture. Ciborra part ici du principe que l'usager est un étranger car les concepteurs des TIC ne peuvent pas prévoir le comportement des personnes dans leur totalité, dans la mesure où ces dernières ont des comportements évolutifs, et qu'on ne peut qu'imaginer les besoins futurs et les caractéristiques basiques propres à la majorité des usagers. Il faut donc autoriser l'étranger (l'usager) à une certaine marge de manœuvre au cours de l'usage. Cela lui permet de «jouer » notamment sur les fonctionnalités des outils lors de l'implémentation des systèmes.

L'enquête menée a confirmé nos hypothèses et mis l'accent sur le fait que le bricolage est une phase nécessaire, et toujours d'actualité, de l'appropriation des technologies dans le contexte de l'entreprise. Il ne s'agit pas uniquement de «faire sien» un système d'information, mais il s'agit d'adopter un comportement qui permet aux usagers de modifier de façon régulière et permanente le dispositif avec lequel les professionnels travaillent. Cet apprentissage permet l'acquisition d'une vraie compétence : celle de bricoler ( l'art de faire ») pour adapter les systèmes d'information à des besoins en continuelle évolution. Le bricolage donne ainsi la possibilité aux usagers de mettre en pratique une certaine autonomie garante de leur efficacité et de leur adéquation au poste.

Au-delà de l'acquisition des nouvelles compétences et du maintien du niveau d'efficacité au travail, il semble que cette façon de faire soit issue d'un consensus implicite entre les usagers et le fonctionnement de l'organisation : cette dernière, pour répondre à son besoin de compétitivité (et compte tenu de ses impératifs financiers, notamment) ne peut que laisser faire les usagers 
En d'autres mots, le bricolage peut permettre, à un acteur individuel, d'améliorer la qualité de son utilisation des TIC et de devenir par la suite porteur d'usage social.

- Le second point met l'accent sur la nécessité d'apprécier cette action humaine qu'est le bricolage au regard du contexte dans lequel elle se trouve. Le bricolage correspond à une action située qui ne prend sens qu'au travers des différents éléments du système organisationnel complexe qui motivent sa mise en œuvre. Le bricolage des TIC pour une meilleure appropriation trouve ainsi sa signification d'efficacité dans le contexte qui la légitime. Cela dit, cette action devient une obligation, une démarche incontournable, une nécessité pour les acteurs, au moins à un certain niveau. Ainsi, n'y aurait-il pas à penser l'appropriation des SI à travers le développement d'une capacité à s'adapter au système, à apprendre à être agile? Cela pose toute la question de la formation à cette capacité si particulière que bien des usagers nomment "la souplesse", "l'adaptabilité ». Certaines entreprises développent cette capacité au travers de ce qui s'appelle «le management agile » (nous soulignons ici que ce type de formation s'adresse au cadre moyen et supérieur, ce qui est en corrélation avec les résultats que nous avons obtenus dans notre enquête) (Barrand, 2006). L'agilité se décline au travers de 3 axes essentiels.

- Le premier demande une motivation non seulement personnelle (nécessité d'atteinte des objectifs individuels par exemple) mais également et surtout collective (obligation d'aider l'entreprise à tirer son épingle du jeu par exemple). Cette dernière permet de créer une dynamique et de trouver des solutions pratiques aux problèmes de terrain.

- Le second axe utilise les TIC et les SI pour créer de la valeur ajoutée. Chaque outil est personnalisé en fonction du besoin professionnel. L'objectif est de permettre une réponse innovante et adaptée à l'organisation. Mais, du même coup, les systèmes évoluent en permanence et ne se stabilisent que très peu. Dans la perspective du management agile, il faut donc optimiser les supports informatiques de façon à répondre à l'évolution de l'organisation, et non plus seulement à suivre les innovations du marché.

- Le troisième point souligne la nécessité d'avoir décrit des procédures issues de l'acteur collectif et éprouvées par lui. Dans ces conditions seulement, l'acteur collectif sera en mesure d'en pallier les dysfonctionnements.

In fine, le management agile permet d'améliorer la maîtrise des coûts et de l'adaptation face aux exigences nouvelles des clients, par l'innovation permanente, pour ne plus subir son environnement, mais précéder les changements issus de l'acteur collectif.

$\mathrm{Au}$ regard des approches structurationnistes et de précédents travaux sur l'usage des TIC au sein des réseaux d'acteurs professionnels (Comtet, 1999), nous soulignons une fois encore la nécessité pour la compréhension des problématiques organisationnelles actuelles de prendre en compte les théories de l'action. Dans l'approfondissement d'un objet d'étude centré sur l'interaction sociale, les théories de l'action viennent compléter l'étayage théorique structurationniste par rapport à l'appropriation et aux interactions. Elles soulignent l'intérêt d'une approche conjointe de l'action et de la connaissance fondée sur les processus d'interaction.

Dans une visée plus managériale, il semble essentiel de ne pas refreiner toutes velléités de transformation de la technologie, (notamment dans la phase d'implémentation mais pas uniquement), mais de l'envisager différemment, dans la mesure où cette dernière est le fruit de l'interaction entre l'acteur et le dispositif : son efficacité d'usage dépendrait donc des modifications, des améliorations, que la technologie « subit » de la part des acteurs de telle sorte qu'elle devient le produit d'un arrangement tacite entre l'organisation et l'acteur. 


\section{BIBLIOGRAPHIE}

Benghozi, P. J., (2002), "Technologie et organisation : le hasard et la nécessité", Annales des Télécommunications, mars-avril, pp. 289-305.

Barrand, J., (2006): Le manager agile, Vers un nouveau management pour affronter la turbulence, Collection Progrès du management, Dunod, Paris

Ciborra, C., (2004), Bricolage, heuristics, serendipity, and make-do, in The Social Study of Information and Communication Technology: innovation, actors and contexts, Oxford University Press, p.19

Ciborra, C., (2004), Problem solving, The Social Study of Information and Communication Technology: innovation, actors and contexts, Oxford University Press, p 25.

Clark, J., Modgil, C., Modgil, S., (1990), Anthony Giddens : consensus and contreversy. eds Anthony Giddens, Consensus \& controversy, Falmer press, New york, p352.

Comtet, I.., (1999), Les acteurs professionnels en réseau de communications, Thèse de doctorat, Université Paris Panthéon-Assas.

De Certeau, M., (1990), L'invention du quotidien. 1. Arts de faire. Paris, Gallimard

De Sanctis, G., Poole, M.S., (1994), “Capturing the complexity in advanced technology use: adaptive theory". Ed Science The institute of management. Vol 5 Organization science, p121-147

De Vaujany, F.X., (2000), « Technologie perturbatrice, technologie neutre et technologie régénérante : construction et approfondissement de trois archétypes technologiques ", 5 ième Colloque de l'AIM, 8-10 Novembre.

Deltour, F., Vaast, E., (2000), « Quant technologie et organisation construisent un réseau d'échanges professionnels ; une étude de cas structurationniste ", $5^{\text {ième }}$ Colloque de l'AIM, 8-10 novembre

Duymedjian, R., Ruling, C.C., (2005), « Le manager bricoleur : essai de construction d'une image légitime », Moderniser la gestion des hommes, Editions liaisons sociales, Chapitre 7, Paris.

Fisette, D., Livet, P., (1993), L'action mise en cause, Les théories de l'action aujourd'hui, PUF, Paris.

Giddens, A., (1984), The constitution of society: outline of the theory of structuration, Berkerley, University of California Press.

Lévi-strauss, C., (1962), La pensée sauvage, Paris, Plon, p.31

Mayere, A., (2003), « Rationalisation de la communication et organisations contemporaines : le cas de projets d'implantations de projets PGI/ERP », Communication et Organisation, Ed Université M. Montaigne, Bordeaux.

Orlikowski, W. J., (2000), "Using technology and constituting structures: a practice lens for studying technology in organizations”. Organization Science, pp404-428

Orlikowski, W.J., (1992), “The duality of technology: rethinking the concept of technology in organisations". Organization science, vol 3, n³, p 406.

Orlikowski, W.J., (1996), "Improvazing organizational transformation overtime : a situated change perspective". Information System research, vol 7, $\mathrm{n}^{\circ} 1$. 


\section{RÉSUMÉS}

Dans le contexte de la mise en pratique de TIC, les acteurs «bricolent » souvent un usage prescrit de façon à ce que ce dernier soit efficace en fonction de l'objectif qu'on lui aura assigné. On fait ici appel à la notion de bricolage comme phase de l'appropriation. De ce fait, l'appropriation est réalisée mais détournée en fonction la relation existant entre les usages des TIC (pour une activité professionnelle) et les structures d'usages (entreprises). Cette appropriation impose une réelle relation entre capacité au bricolage et compétence adaptative.

In the context of implementation of TIC, the actors often arrange or "bricolent" a prescribed use according to the objective assigned. Here, the concept of "bricolage" is considered like phase of the appropriation. So the appropriation is achieved but diverted in function of the relation existing between the uses of the TIC and the structures of uses. This appropriation needs a real deal between "bricolage" and adaptative skill.

\section{INDEX}

Mots-clés : appropriation, bricolage, structurationnisme, compétence professionnelle

Keywords : appropriation, bricolage, structurationnism, uses of TIC, adaptative skill

\section{AUTEUR}

\section{ISABELLE COMTET}

Isabelle Comtet est actuellement maître de conférences en Sciences de l'Information et de la Communication à l'IUT Lyon III, chercheur au Laboratoire Sicomor de l'IAE de Lyon et vacataire à Grenoble Ecole de Management. Ses thèmes de recherches sont principalement centrés sur l'évolution des pratiques des TIC dans les entreprises, en lien avec le management et les ressources humaines. L'objectif est de traduire en termes d'approche méso les changements de l'activité professionnelle induits par l'usage, contraint ou non, des TIC au quotidien.

isabelle.comtet@univ-lyon3.fr 